\title{
Vertrauen statt Kontrolle
}

Steile Hierarchien und enge Kontrollen sind längst nicht mehr zeitgemäß, doch in Kliniken nach wie vor gang und gäbe. Die RoMed Kliniken in Bayern gehen aber einen anderen Weg, hin zu flachen Strukturen und einer selbstorganisierten Führung.

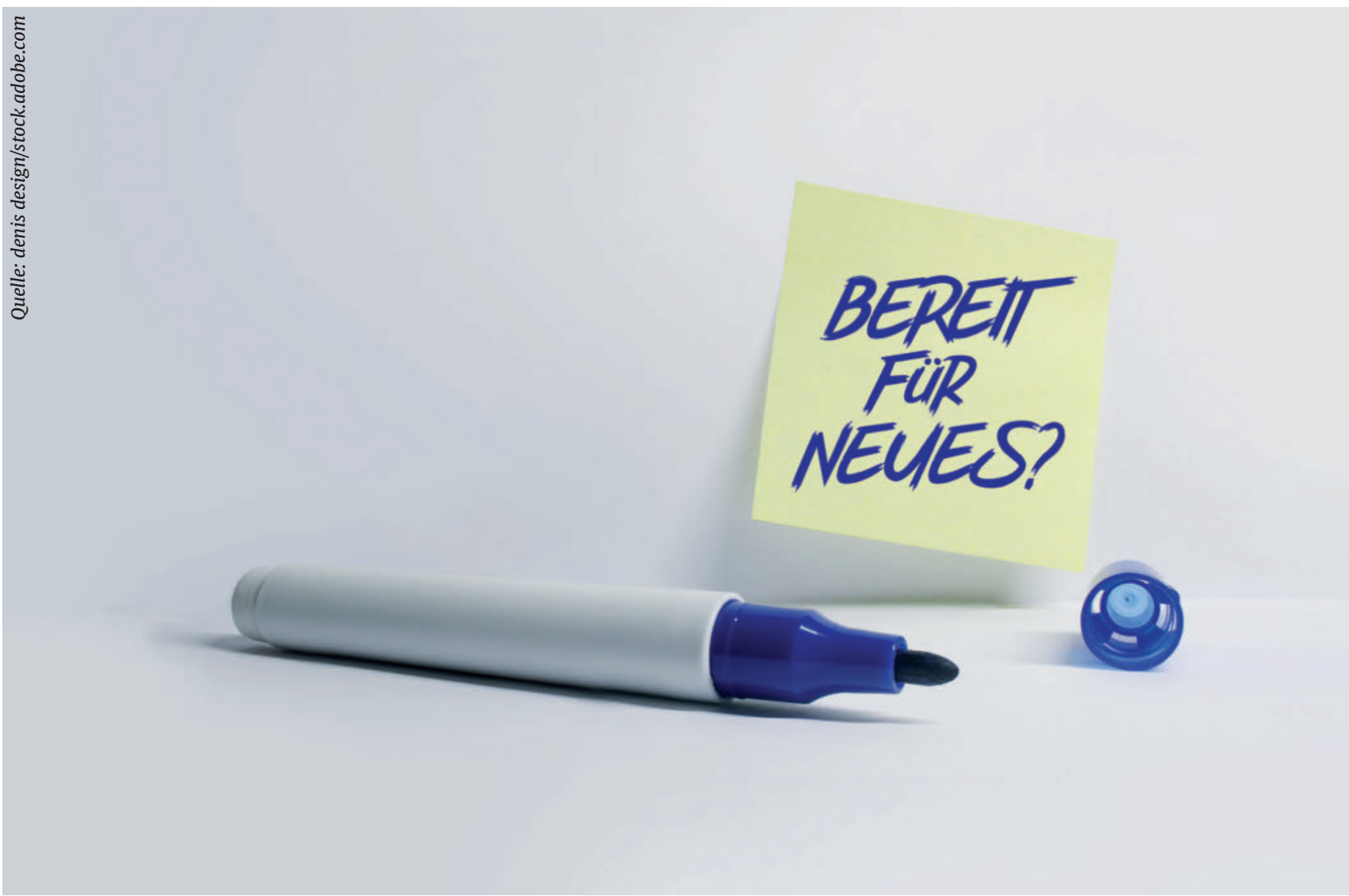

- Zu Beginn des 20.Jahrhunderts entstand die starre Krankenhaushierarchie, die bis heute in den deutschen Kliniken zu spüren ist. Die Folge ist mittlerweile Frustration, innere Kündigungen, hohe Fluktuation und Qualitätsmängel in der patientenorientierten Versorgung. Um dieser Situation entgegenzuwirken, entwickeln die RoMed Kliniken innovative Arbeitsbedingungen im Gesundheitswesen. Flache Hierarchien, Wertschätzung und Freiräume für die Mit- arbeiterinnen und Mitarbeiter sollen in der Unternehmenskultur fest verankert werden, das ist das Ziel. Mit Hilfe von New Work möchte man diesen Gedanken weitertragen.

\section{Mehr Freiheit und Selbstständigkeit}

Die Bedeutung, das Konzept und der Begriff von New Work lassen sich auf das Jahr 1980 zurückführen. Damals hatte der österreichisch-US-amerikanische Sozialphilosoph
Fritjof Bergmann im Zuge der Automatisierung der Automobilunternehmen in den USA erstmals das Thema New Work aufgegriffen und in diesem Zusammenhang auch die „Neuen Kulturen“. Dabei drehten sich seine Forschungen in erster Linie um die Kernfragen „Wie der Mensch denn Freiheit erlangen kann?“ „Und wie die Sinnhaftigkeit im Zusammenhang mit seiner Arbeitswelt?“ Auf dieser Ausgangsbasis entwickelten sich schließlich die Ideen zu New Work, der 
Bewegung der „Neuen Arbeit“. Bergmann ging davon aus, dass die neuen Technologien den Menschen ermöglichten, sich aus der Unterdrückung der Lohnarbeit zu lösen und an ihrer Stelle die Tätigkeit zu wählen, die sie wirklich erfüllt. Und dies mit mehr Freiheit und Selbstständigkeit unter den auf sie zugeschnittenen Umständen. Dass der Mensch nur eintönige, immer wiederkehrende Fließbandarbeit tätigt, sei seiner Ansicht nach nicht sinnstiftend. Der Psychologe Markus Väth, der Co-Founder des „New Work“-Think Tanks „humanfy“ ist, geht hier noch einen Schritt weiter: Er argumentiert, dass mit den Möglichkeiten der neuen digitalen Techniken, die die Grenze zwischen Arbeit und Privatleben aufheben, auch der Begriff „Work-Life-Balance“ seine Bedeutung verliert. Aber alles der Reihe nach.

\section{Was genau verbirgt sich hinter New Work?}

New Work ist ein fundamentaler Systemwechsel, der sich intensiv mit der Arbeitswelt der Zukunft beschäftigt. Hierbei stehen die Förderung der Autonomie und die Selbstbestimmung der Mitarbeiterinnen und Mitarbeiter im Fokus. Ziel ist es, die Persönlichkeit der Beschäftigten zu stärken und ihnen größere Entwicklungs- und Entscheidungskompetenzen zu ermöglichen. Veränderungen in den Strukturen und im Führungsstil sind für diesen Wandel ebenso unverzichtbar wie eine kontinuierliche Reflexion des eigenen Führungsverhaltens. Das Thema New Work im Gesundheitswesen hat bisher in Deutschland nur wenig Anklang gefunden. Nur vereinzelt sind kleinste Berührungspunkte mit New Work im Gesundheitswesen zu beobachten. Lohnenswert ist deshalb ein Blick ins Ausland. Wie New Work in der Praxis aussehen kann, zeigt beispielsweise Buurtzog, ein Pflegedienst aus den Niederlanden,

Tab. 1 Komplizierte und komplexe Strukturen - eine Gegenüberstellung

\begin{tabular}{l|l}
\hline Komplizierte Strukturen & Komplexe Strukturen \\
\hline Regeln & Prinzipien \\
\hline Starre Systeme & Soziale Systeme \\
\hline Leitfrage: Wie geht es? & Leitfrage: Wer kann damit umgehen? \\
\hline Währung: Wissen & Währung: Können \\
\hline
\end{tabular}

der sich in kleinen Teams selbst organisiert - eine Pflegende schreibt zum Beispiel die Dienstpläne, eine andere ist etwa für die Akquise und Einstellung von Mitarbeitern zuständig. Dass das Konzept aufgeht, zeigt auch der starke Zulauf an Bewerbern, die bei Buurtzorg arbeiten wollen. Und auch in Schweden gibt es Pilotprojekte für diverse Kranken-, Pflege- und Sozialeinrichtungen, wobei versucht wird, ein New Workartiges Verhalten beziehungsweise einzelne Elemente daraus zu etablieren. Auch die gemeinnützige Organisation RHD aus den USA setzt sich erstmals für ein neu definiertes Management im Gesundheitswesen und im Sozialen ein. In deutschen Krankenhäusern oder Unternehmen hingegen sind aktuell nur sehr wenige Ansätze für ein Etablieren von New Work im Gesundheitswesen zu finden.

\section{Die Zeichen der Zeit erkennen}

Doch die starre Krankenhaushierarchie ist in der heutigen Arbeitswelt einfach nicht mehr zeitgemäß. Die Erwartungen der Mitarbeiterinnen und Mitarbeiter an ihren Arbeitgeber führen zu einer Veränderung auf dem Arbeitsmarkt. Diesem Umstand müssen die Kliniken in ihrer Personalakquise und in der Bindung ihrer Mitarbeiterinnen und Mitarbeiter Rechnung tragen. Die Führungskräfte der RoMed Kliniken haben die Zeichen der Zeit erkannt und wollen mit Hilfe eines strukturellen Wandels die Zukunft der Arbeit neu gestalten. Mit einer Unternehmenskultur der Mitarbeiter- wertschöpfung und dem Ermöglichen von individueller Entfaltung der Beschäftigten wollen sich die RoMed Kliniken als Magnetkrankenhaus für ausgezeichnete Mitarbeiter- und Kundenzufriedenheit in Bayern etablieren. Hierfür ist es entscheidend, im Krankenhaus-Setting zwischen komplizierten und komplexen Strukturen zu differenzieren. Komplizierte Strukturen sind starre Reglements, die der qualitativen Versorgung der Patientinnen und Patienten dienen. Im Gegensatz dazu sind die komplexen Strukturen durch prinzipielle Vorgehensweisen gekennzeichnet. Auf dieser Grundlage sind hier Abweichungen durchaus möglich und erwünscht.

Die einzelnen Punkte unserer Zielsetzung:

- Wertschöpfung im Mittelpunkt

- Kundenbedürfnissen auf direktem Weg nachkommen

- Beschäftigung vermeiden, Arbeit erhöhen

- Gefühl von Wirksamkeit steigern

- Sinngefühl vermitteln

- Mitverantwortung übertragen

- Wirtschaftliches Handeln

- Unternehmenskultur verändern

Im ersten Schritt beschäftigen sich die Führungskräfte auch mit den Strukturen im Unternehmen, die bisher durch Kompliziertheit geprägt waren. Ziel ist es, diese, wo möglich, zu reduzieren und den Fokus auf die Komplexität zu richten. 


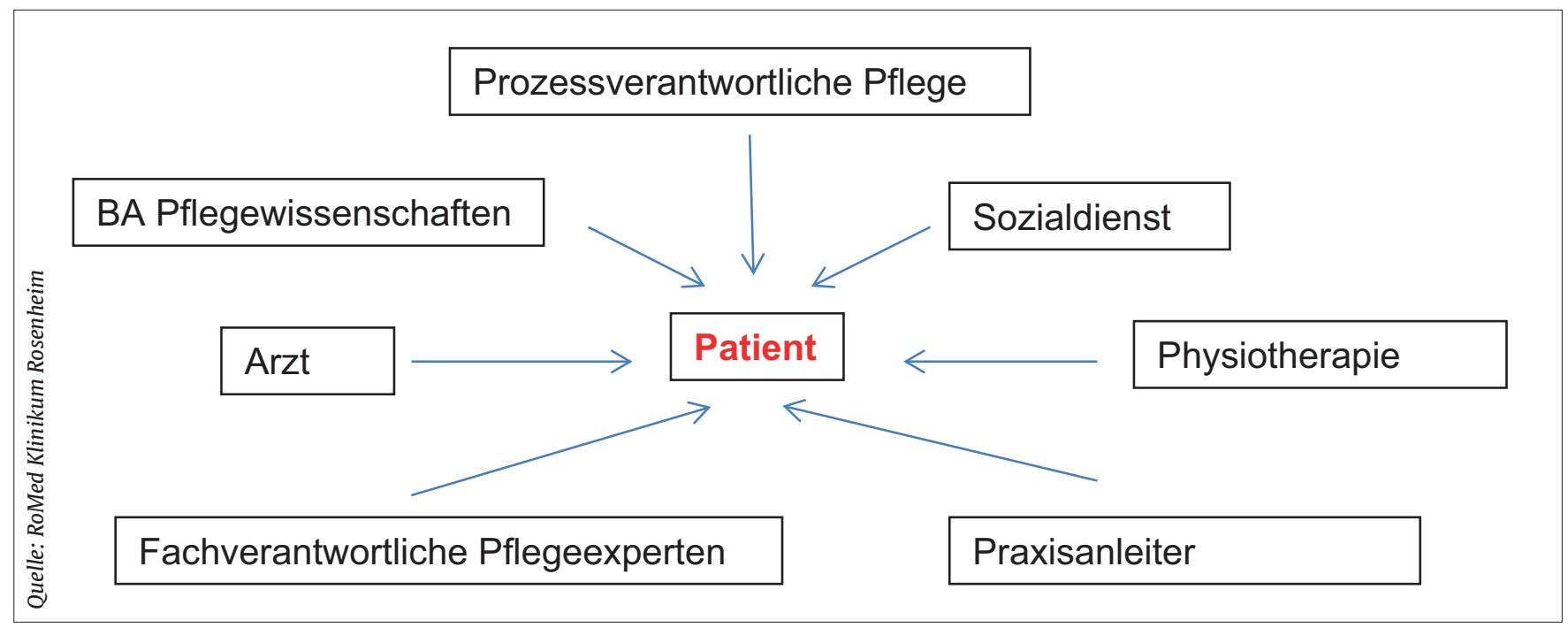

Abb. 1 Geplante Struktur der Stationen und Funktionen in den RoMed Kliniken

Daraus entwickeln sich die 7 Prinzipien von „New Work“

1. Den Markt ins Unternehmen lassen (absoluter Fokus auf den „Nutzer“)

2. Mannschaften bauen

3. Dezentrale selbstorganisierte Führung

4. Als Team fungieren

5. Selber denken lassen

6. Wissen demokratisieren

7. Fit halten

\section{Macht abgeben, Vertrauen aufbauen}

Die Umsetzung der 7 Prinzipien des New Work in den RoMed Kliniken erfolgt in enger Zusammenarbeit mit den Beschäftigten. Denn: Den New-Work-Gedanken mit Leben füllen, können nur die Mitarbeiterinnen und Mitarbeiter der RoMed Kliniken selbst. Seinen Anfang nehmen, muss das Umdenken jedoch in der Führung. Dieser Prozess begann vor gut einem Jahr in der Pflegedirektion mit einem ganztägigen Auftaktworkshop. Teilnehmer waren Pflegedienstleitungen, Ärzte, die Leitung aus der Personalabteilung und dem Marketing, aber auch Trainees und studentische Hilfskräfte. Um den neuen Weg zu beschreiten, muss allen Teilnehmern klar sein, dass das Prinzip Führung neu definiert werden muss. Das Motto lautet: Abgabe von Macht und Kontrolle - Verantwortungsdelegation und Aufbau von Vertrauen! Führungskräfte, die bislang über Qualifizierungsmaßnahmen und Sozialisation damit nie in Berührung gekommen sind, müssen ihre Einstellung und ihr Verhalten entsprechend ändern. Aus der Organisationspsychologie ist hinlänglich bekannt, dass dieser Prozess einen längeren Zeitraum einnimmt. Das Entwickeln und Etablieren des New Work-Gedankens in der Führung erfolgt inzwischen in regelmäßigen Ganztagesworkshops bei RoMed bis zum heutigen Tag. Ziel soll es sein, dass die Führungskräfte unter einer nicht vorgebenden, sondern begleitenden Moderation eigenständig Inhalte erarbeiten. Hier ist eine wichtige Erkenntnis, dass besonders auf persönliche Ängste und Unsicherheiten eingegangen werden muss. Im Verlauf entwickelte sich auch die zukünftige Struktur der Stationen und Funktionen im Unterneh- men und damit einhergehend der Begriff „Projekt Musterbrecher“. Jeder Mitarbeiter, der an einem Projekt im Kontext von New Work teilnimmt, erhält diesen Button. Dies soll dazu führen, die jeweiligen Mitarbeiter in ihrem Engagement sichtbar zu machen, damit es auch zu einem internen Austausch kommt.

Ein- bis zweimal die Woche bespricht ein interdisziplinäres Team besonders herausfordernde Patienten und legt dabei ein einheitliches Vorgehen fest. Gleichzeitig werden hier im stationären Kontext Entscheidungen getroffen, Verantwortung übernommen und Innovationen entwickelt. Bekannt ist dieses Vorgehen zum Teil schon aus der Psychiatrie, leider hat es bislang kaum Einzug in die Somatik gehalten.

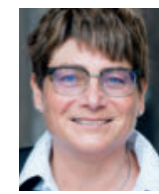

Judith Hantl-Merget ist Pflegedirektorin an den RoMed Kliniken Rosenheim. Co-Autorin: Anna-Maria Rittner. 